\title{
Review of minimal access versus transcatheter aortic valve replacement for patients with severe aortic stenosis
}

\author{
Carson T. Hoffmann, Jacob A. Heiner, Tom C. Nguyen \\ Department of Cardiothoracic and Vascular Surgery, McGovern Medical School, University of Texas at Houston, Memorial Hermann Hospital- \\ Heart and Vascular Institute, Houston, Texas, USA \\ Correspondence to: Tom C. Nguyen, MD. Department of Cardiothoracic and Vascular Surgery, University of Texas, Memorial Hermann, 6400 \\ Fannin Street, Suite 2850, Houston, Texas 77030, USA. Email: tom.c.nguyen@gmail.com.
}

\begin{abstract}
Transcatheter aortic valve replacement (TAVR) and minimally invasive aortic valve replacement (miniAVR) have become alternatives to surgical aortic valve replacement via median sternotomy (SAVR) to treat severe aortic stenosis (AS). Despite increased interest and utilization, few studies have directly compared TAVR and miniAVR. A review of the current literature shows TAVR to be an indispensable tool for inoperable, high-risk, and perhaps intermediate-risk patients with severe AS. However, it is associated with a number of deleterious perioperative outcomes, such as valvular regurgitation and vascular complications. MiniAVR is associated with decreased intensive care unit (ICU) and hospital length of stay, a lower incidence of blood transfusions, decreased ventilation time, and improved cosmetic results. MiniAVR maintains potential advantages over SAVR, including the implantation of a durable prosthesis and low rates of perioperative myocardial infarction and paravalvular leak. It is associated with longer aortic cross clamp and cardiopulmonary bypass (CPB) times; however, the use of sutureless valve implants can circumvent this. Studies comparing TAVR and miniAVR demonstrate decreased postoperative mortality, valvular regurgitation, and incidence of stroke in the miniAVR cohorts. Few studies currently exist comparing TAVR and miniAVR, as it is hard to compare the typically low-risk miniAVR versus high-risk TAVR patient populations. It is clear that both strategies will be cornerstones in the modern AVR era, but the situations in which to apply each strategy have not yet been clearly delineated. This highlights the need for surgeons to adopt these minimally invasive techniques. We believe there is a compelling role for miniAVR in low- and intermediate-risk patients, but due to the paucity of data, neither TAVR nor miniAVR should be discounted before a randomized, risk-stratified trial is performed. More studies are needed to compare TAVR and miniAVR in low- and intermediate-risk patients.
\end{abstract}

Keywords: Aortic stenosis (AS); aortic valve replacement; minimally invasive; transcatheter

Submitted Jul 24, 2017. Accepted for publication Aug 30, 2017.

doi: 10.21037/acs.2017.09.02

View this article at: http://dx.doi.org/10.21037/acs.2017.09.02

\section{Introduction}

Aortic valve replacement (AVR) is the second most common cardiac procedure performed, with aortic stenosis (AS) being the most common valve disease as a result of an aging population (1). A prospective, population-based study found the incidence of AS to be $0.2 \%$ in the 50-59 years cohort, $1.3 \%$ in the $60-69$ years cohort, $3.9 \%$ in the $70-79$ years cohort, and $9.8 \%$ in the $80-89$ years cohort (2).
A survival rate of $50 \%$ at 2 years and $20 \%$ at 5 years after symptom onset has prompted the development of multiple therapeutic strategies (3).

Traditionally, surgical aortic valve replacement via median sternotomy (SAVR) has been the gold standard to treat severe AS, as this approach has been highly successful in providing freedom from structural valve failure rates of $70-90 \%$ at 10 years and $50-80 \%$ at 15 years (4). However, in this era of advancements in minimally invasive 
operations, patients increasingly want the least invasive approach to correct complex problems, assuming that it is safe, as good as the status quo, and potentially cost effective. In recent years, transcatheter aortic valve replacement (TAVR) has emerged as a promising option for high- and potentially intermediate-risk patients, and there are several studies comparing TAVR versus SAVR. The drive towards minimally invasive strategies has also increased the demand and adoption of minimally invasive aortic valve replacement (miniAVR). Nguyen et al. demonstrated an increased growth for both of these techniques between 2011 and 2014 (5). Transfemoral TAVR and miniAVR displayed a $595 \%$ and $57 \%$ increase from 2011 to 2014, respectively (5). Johnston and Roselli reported an increase in miniAVR from $12.4 \%$ to $29.6 \%$ of the total aortic valve procedures at the Cleveland Clinic, from 1996 to 2013 (6).

Despite the increased interest in TAVR and miniAVR, only a handful of studies comparing both approaches exist. The goal of this article is to provide an in-depth review of the current literature comparing TAVR and miniAVR for patients with severe AS.

\section{Results}

\section{TAVR}

One of the major advancements in the management of severe AS came with the introduction of TAVR in 2002. Before this intervention, at least $30 \%$ of patients with severe, symptomatic AS did not undergo SAVR due to comorbidities and risk (7). The Placement of Aortic Transcatheter Valves (PARTNER) 1B trial was the first randomized clinical trial comparing TAVR $v s$ standard therapy in 358 high-risk [mean Society of Thoracic Surgeons Predicted Risk of Mortality (STS PROM) of $11.6 \%]$, inoperable patients with severe AS. TAVR was shown to be superior to the standard therapy of medical management and balloon aortic valvuloplasty, with a reduced 1-year all-cause mortality (30.7\% vs. 49.7\%; $\mathrm{P}<0.001)(8)$. The trial revealed lower rates of cardiac symptoms in the TAVR cohort. Of the surviving patients, $74.8 \%$ in the TAVR cohort $v s .42 \%$ who received standard therapy were asymptomatic or had mild symptoms (New York Heart Association class I or II) at 1 year $(\mathrm{P}<0.001)(8)$.

The PARTNER 1A study was a randomized trial comparing TAVR vs. SAVR in 699 high-risk (mean STS PROM of $11.8 \%$ ), operable patients. The study showed comparable mortality rates at 30 days $(3.4 \%$ vs. $6.5 \%$;
$\mathrm{P}=0.07)$ and 1 year $(24.2 \%$ vs. $26.8 \% ; \mathrm{P}=0.44)$ (9). The rate of all neurologic events (strokes and transient ischemic attacks) was increased in the TAVR cohort at 30 days (5.5\% vs. $2.4 \% ; \mathrm{P}=0.04)$ and at 1 year $(8.3 \%$ vs. $4.3 \% ; \mathrm{P}=0.04)(9)$. The 5 -year outcomes were comparable with regard to all-cause mortality $(67.8 \%$ vs. $62.4 \% ; \mathrm{P}=0.76)$ and all neurologic events (15.9\% vs. $14.7 \% ; \mathrm{P}=0.35)$ (10).

The PARTNER 2A trial investigated the use of TAVR in intermediate-risk patients (STS PROM 4-8\%) with severe AS. Patients were randomized to either TAVR (with transfemoral and transapical cohorts) or SAVR. No significant difference in all-cause mortality or disabling stroke at 2 years between the TAVR and SAVR groups was found [hazard ratio $(\mathrm{HR})=0.89 ; 95 \%$ confidence interval (CI), 0.73 to $1.09 ; \mathrm{P}=0.25$ ] (11). In the transfemoral-access cohort, TAVR resulted in a lower rate of all-cause mortality and disabling strokes (HR $=0.79 ; 95 \% \mathrm{CI}, 0.62$ to 1.00 ; $\mathrm{P}=0.05)$ (11). Major vascular complications were more frequent in the TAVR group ( $7.9 \%$ vs. $5.0 \% ; \mathrm{P}=0.008)(11)$. Other complications were less frequent in the TAVR group than in the surgery group, including life-threatening bleeding $(10.4 \%$ vs. $43.4 \% ; \mathrm{P}<0.001)$, acute kidney injury ( $1.3 \%$ vs. $3.1 \% ; \mathrm{P}=0.006$ ), and new-onset atrial fibrillation (9.1\% vs. $26.4 \% ; \mathrm{P}<0.001)$ (11).

Thourani et al. conducted a propensity score analysis comparing outcomes at 1 year between intermediaterisk patients who received transfemoral TAVR $v s$. the intermediate-risk patients in the PARTNER 2A study who received SAVR. They showed superiority of TAVR when using the $3^{\text {rd }}$ generation SAPIEN 3 valve (Edwards Lifesciences, Irvine, CA, USA) over SAVR in terms of reduced mortality (7.4\% vs. $13 \%)$, stroke (4.6\% vs. $8.2 \%)$, rehospitalization (11.4\% vs. $15.1 \%)$, and new-onset atrial fibrillation (5.9\% vs. 29.2\%) at 1 year (12).

\section{MiniAVR}

A second option for minimally invasive AVR is miniAVR. This is typically performed via right anterior thoracotomy or ministernotomy in lieu of a full median sternotomy. This reduces the incision from an average of 24.5 to $7.17 \mathrm{~cm}$ with a ministernotomy, or a $5 \mathrm{~cm}$ incision with a thoracotomy $(13,14)$. MiniAVR has been shown to: reduce length of intensive care unit (ICU) and hospital stay, reduce ventilation time, decrease the need for blood transfusion, decrease pain, and improve the cosmetic result (15-17).

A Cochrane review that included seven randomized controlled trials compared AVR via median sternotomy $v$ s. 
ministernotomy. It illustrated no effect of ministernotomy on mortality [risk ratio $(\mathrm{RR})=1.01 ; 95 \% \mathrm{CI}, 0.36$ to 2.82] (18). No increase in aortic cross-clamp (AXC) or cardiopulmonary bypass (CPB) time was found. There was no difference in length of hospital stay.

Phan et al. published a meta-analysis comparing miniAVR $v s$. SAVR. The analysis included 50 studies with 12,786 patients. They reported increased AXC (weighted mean difference of 8.09 minutes; $\mathrm{P}<0.00001$ ) and CPB times (weighted mean difference of 8.16 minutes; $\mathrm{P}<0.0001)$ with miniAVR (19). The miniAVR cohort was associated with lower rates of perioperative mortality ( $1.9 \%$ vs. $3.3 \% ; \mathrm{P}=0.02$ ), perioperative transfusion $(36 \%$ vs. $52.4 \% ; \mathrm{P}=0.001)$, renal failure ( $2.5 \%$ vs. $4.2 \% ; \mathrm{P}=0.04)$, and length of stay in the ICU (weighted mean difference of -0.6 days) and hospital ( -1.34 days; $\mathrm{P}=0.0007)$ (19). The two cohorts showed no significant difference in rates of neurologic events, atrial fibrillation, pacemaker implants, and myocardial infarctions (19).

\section{MiniAVR via thoracotomy $v s$. ministernotomy}

When discussing miniAVR, it is important to differentiate between ministernotomy and right minithoracotomy. Miceli et al. reviewed 406 patients who underwent miniAVR with either a right minithoracotomy or ministernotomy. The two groups averaged similar AXC (89.7 vs. 84.3 minutes; $\mathrm{P}=0.07$ ) and $\mathrm{CPB}$ times (124.9 vs. 122.2 minutes; $\mathrm{P}=0.48)(15)$. Overall in-hospital mortality was $1.2 \%$ with no significant difference between the cohorts (15). The minithoracotomy group experienced a lower incidence of postoperative atrial fibrillation ( $19.5 \%$ vs. $34.2 \%$; $\mathrm{P}=0.01)$, shorter ventilation time (median, 7 vs. 8 hours; $\mathrm{P}=0.003$ ), shorter ICU length of stay (median, 1 day; interquartile range, $1-1$; vs. median, 1 day; interquartile range, $1-2 ; \mathrm{P}=0.001$ ), and ward stay (median, 5 vs. 6 days; $\mathrm{P}=0.0001)$ (15). No difference in rate was found for postoperative stroke, re-exploration for bleeding, or blood transfusion. The survival rate in the minithoracotomy $v s$. ministernotomy group at 1 year was $97 \%$ vs. $86 \%$ and $94 \%$ vs. $80 \%$ at 5 years, respectively $(\mathrm{P}=0.1)(15)$.

Fattouch et al. performed another comparison of miniAVR via minithoracotomy or ministernotomy. Inhospital mortality and death within 30 days of the operation were comparable between both cohorts $(1.1 \%$ in the minithoracotomy cohort $v s .3 .3 \%$ in the ministernotomy cohort; $\mathrm{P}=0.28)$ (20). AXC (62.6 vs. 62.4 minutes; $\mathrm{P}=0.11)$ and $\mathrm{CPB}$ times (78.7 vs. 76.8 minutes; $\mathrm{P}=0.64$ ) were comparable between the minithoracotomy and ministernotomy groups, respectively (20).

\section{TAVR $v s$. miniAVR}

Few studies have directly compared TAVR and miniAVR. Miceli et al. retrospectively analyzed patients with severe AS who underwent either TAVR, via transapical or transfemoral approach, or miniAVR via a right thoracotomy with a sutureless valve. After propensity score analysis, 37 matched pairs were compared. The in-hospital mortality rate was $8.1 \%$ in the TAVR group and $0 \%$ in the miniAVR group $(\mathrm{P}=0.25)$ (21). Rates of stroke and transient ischemic attack were $5.4 \%$ and $2.7 \%(\mathrm{P}=0.3)$ in the TAVR group, respective to the approaches noted above, with none occurring in the miniAVR group at a median followup period of 13 months (21). Although not statistically significant, the survival rates were higher in the miniAVR cohort compared to TAVR ( $91.6 \%$ vs. $78.6 \%$ at 1 year and $91.6 \%$ vs. $66.2 \%$ at 2 years, respectively (HR $=0.7 ; 95 \%$ CI, 0.7 to $49.8 ; \mathrm{P}=0.1)$ ) (21). The TAVR cohort demonstrated higher rates of mild and moderate paravalvular leak $37.8 \%$ and $27 \%$, respectively) compared to the miniAVR cohort (2.7\% mild and $0 \%$ moderate) $(\mathrm{P}<0.001)(21)$.

Santarpino et al. reported similar results with a retrospective, propensity score analysis comparing TAVR $v s$. miniAVR with a sutureless valve in high-risk patients. They report an incidence of aortic regurgitation of $13.5 \%$ in the TAVR group $v s .0 \%$ in the miniAVR group $(\mathrm{P}=0.27)$ and a cumulative survival of $86.5 \%$ vs. $97.3 \%(\mathrm{P}=0.015)$ in the TAVR and miniAVR cohorts respectively, with a mean follow-up of 18.9 months (22).

Terwelp et al. performed a propensity score analysis comparing SAVR, TAVR and miniAVR (23). Analysis of the matched transfemoral TAVR and miniAVR pairs demonstrated no difference in 30-day mortality. Transfemoral TAVR was associated with a higher postoperative stroke rate compared to miniAVR $(3.6 \%$ vs. $0.4 \% ; \mathrm{P}=0.02)$, but a lower incidence of postoperative atrial fibrillation ( $4 \%$ vs. $19.4 \%$; $\mathrm{P}<0.01)(23)$.

\section{Discussion}

The emergence of TAVR has offered a means to treat inoperable patients with severe AS, with its use quickly expanding to high-risk patients and future applications in sight for intermediate- and low-risk patients. The ability to replace the aortic valve via a small groin incision is a highly 
attractive option to patients, compared to a full sternotomy. However, it has been demonstrated that TAVR is associated with increased perioperative complications, including: stroke or transient ischemic attack, incidence of vascular complications, permanent pacemaker implantation, and postoperative moderate and severe para- and transvalvular regurgitation (24). No difference in all-cause mortality has been identified between SAVR and TAVR $(9,10,24,25)$. MiniAVR is a second option for minimally invasive management of AS and has been shown to be equivalent to and possibly better than SAVR in terms of morbidity and mortality, while decreasing blood loss, ICU and hospital length of stays and recovery time $(13,19,26-28)$. The main drawback is the technical difficulty and prolonged AXC and CPB times. However, the utilization of sutureless valve implants has been shown to circumvent both of these pitfalls $(21,22,26,29,30)$. Although miniAVR is more invasive than TAVR, it retains the advantages inherent to SAVR. This includes the insertion of a durable prosthesis, rates of paravalvular leak of $<1 \%$, and comparable rates of perioperative myocardial infarction of $0.4 \%$ with miniAVR vs. $0.7 \%$ with SAVR ( $\mathrm{P}=0.77)(19,31)$.

A paucity of data exist comparing TAVR and miniAVR. Direct studies are difficult as TAVR patients have typically been high-risk, while miniAVR patients tend to be lowrisk, as it is associated with longer $\mathrm{AXC}$ and $\mathrm{CPB}$ times. Even with propensity score analysis, it is difficult to make comparisons between the two techniques.

Both Miceli and Santarpino demonstrated a reduced survival rate and increased aortic regurgitation in the TAVR cohort $(21,22)$. Terwelp demonstrated differences in postoperative rates of stroke and atrial fibrillation between TAVR and miniAVR. Therefore, it is important to consider the risk and impact of these complications on the individual patient when choosing between the two (23). The fact that miniAVR has been shown to be equivalent to SAVR in terms of mortality, coupled with decreased blood loss, ventilator time, and ICU and hospital length of stay, may make miniAVR (especially with a sutureless valve) the ideal solution for managing high-risk patients who are not ideal SAVR candidates. However, it is most likely a first-line option for low- and intermediate-risk patients (26).

Just as variable approaches exist for TAVR (transapical $v s$. transfemoral), miniAVR too can be performed via a number of incision pathways (ministernotomy, minithoracotomy, etc.). Miceli established a higher survival rate at 1 and 5 years and a lower incidence of postoperative atrial fibrillation, shorter ventilation time, decreased ICU and hospital length of stay in the minithoracotomy group as compared to the ministernotomy group (15). These differences demonstrate the need for future studies to separate and distinguish the approaches, in order to more accurately measure and compare miniAVR outcomes.

Considering the current data and literature, we put forth the following opinion for AVR in patients with severe AS; it is clear that TAVR is an indispensable tool for treating severe AS in inoperable patients (8). In high-risk patients, the data appears to support TAVR, and we believe this is an important therapeutic option. The data is less clear for intermediate-risk patients, but from the PARTNER 2A trial and a study by Reardon et al., comparing SAVR $v s$. TAVR in intermediate-risk patients, it appears that transfemoral TAVR is favorable $(11,32)$. We believe it is important to recognize the potential caveats of these trials. Notably, the average age and STS PROM score for patients in PARTNER 2A were 81 years and 5.8\%, respectively (11). Therefore, when evaluating patients for TAVR who are younger, or with a lower STS score, there needs to be recognition that these patients lie outside the demographic of the intermediate-risk trials. The PARTNER 2A study is also limited, in that patients were randomized to TAVR (transfemoral or transapical) or SAVR after being screened to see if the transfemoral approach would be feasible. This represents a selection bias by not randomizing treatment arms and selecting which patients will do better with the transfemoral approach. Another important consideration is that these trials never directly compared TAVR to miniAVR in intermediate-risk patients, and we believe that miniAVR may prove just as efficacious, if not superior.

Without a doubt, TAVR and miniAVR are here to stay. They have both proven to be essential tools that cardiac surgeons need to be comfortable using. With advances in technology and surgical technique, coupled with increased patient demand for minimally invasive procedures, increasing implementation is expected. Thus, it is imperative that more studies be conducted in order to accurately understand and appropriately apply TAVR and miniAVR. Currently, there is no risk-stratified trial directly comparing TAVR and miniAVR and neither technique should be discounted before such a trial is performed.

In conclusion, TAVR and miniAVR are burgeoning options but with little data to compare them. However, we believe there is a compelling role for miniAVR in low- and intermediate-risk patients. In the modern surgical era, even cardiothoracic surgery is beginning to experience the shift towards minimally invasive procedures. There needs to be 
randomized, risk-stratified trials comparing TAVR and miniAVR before well-informed guidelines can be created. Either way, cardiac surgeons need to adopt these techniques as the data and outcomes clearly indicate their usefulness and necessity.

\section{Acknowledgements}

None.

\section{Footnote}

Conflicts of Interest: TC Nguyen is a consultant for Edwards Lifesciences and St. Jude Medical; the other authors have no conflicts of interest to declare.

\section{References}

1. Al-Adhami A, Al-Attar N. Recent advances in aortic valve replacement for aortic stenosis. F1000Research 2016, 5:2542.

2. Eveborn GW, Schirmer H, Heggelund G, et al. The evolving epidemiology of valvular aortic stenosis. the Tromsø study. Heart 2013;99:396-400.

3. Otto CM. Timing of aortic valve surgery. Heart 2000;84:211-8.

4. Kirtane AJ, Leon MB. The Placement of Aortic Transcatheter Valve (PARTNER) trial: clinical trialist perspective. Circulation 2012;125:3229-32.

5. Nguyen TC, Terwelp MD, Thourani VH, et al. Clinical trends in surgical, minimally invasive and transcatheter aortic valve replacement†. Eur J Cardiothorac Surg 2017;51:1086-92.

6. Johnston DR, Roselli EE. Minimally invasive aortic valve surgery: Cleveland Clinic experience. Ann Cardiothorac Surg 2015;4:140-7.

7. Iung B, Cachier A, Baron G, et al. Decision-making in elderly patients with severe aortic stenosis: why are so many denied surgery? Eur Heart J 2005;26:2714-20.

8. Leon MB, Smith CR, Mack M, et al. Transcatheter aortic-valve implantation for aortic stenosis in patients who cannot undergo surgery. N Engl J Med 2010;363:1597-607.

9. Smith CR, Leon MB, Mack MJ, et al. Transcatheter versus surgical aortic-valve replacement in high-risk patients. $\mathrm{N}$ Engl J Med 2011;364:2187-98.

10. Mack MJ, Leon MB, Smith CR, et al. 5-year outcomes of transcatheter aortic valve replacement or surgical aortic valve replacement for high surgical risk patients with aortic stenosis (PARTNER 1): a randomised controlled trial. Lancet 2015;385:2477-84.

11. Leon MB, Smith CR, Mack MJ, et al. Transcatheter or Surgical Aortic-Valve Replacement in Intermediate-Risk Patients. N Engl J Med 2016;374:1609-20.

12. Thourani VH, Kodali S, Makkar RR, et al. Transcatheter aortic valve replacement versus surgical valve replacement in intermediate-risk patients: a propensity score analysis. Lancet 2016;387:2218-25.

13. Moustafa MA, Abdelsamad AA, Zakaria G, et al. Minimal vs median sternotomy for aortic valve replacement. Asian Cardiovasc Thorac Ann 2007;15:472-5.

14. Plass A, Scheffel H, Alkadhi H, et al. Aortic valve replacement through a minimally invasive approach: preoperative planning, surgical technique, and outcome. Ann Thorac Surg 2009;88:1851-6.

15. Miceli A, Murzi M, Gilmanov D, et al. Minimally invasive aortic valve replacement using right minithoracotomy is associated with better outcomes than ministernotomy. J Thorac Cardiovasc Surg 2014;148:133-7.

16. Brown ML, McKellar SH, Sundt TM, et al.

Ministernotomy versus conventional sternotomy for aortic valve replacement: a systematic review and meta-analysis. J Thorac Cardiovasc Surg 2009;137:670-9.e5.

17. Murtuza B, Pepper JR, Stanbridge RD, et al. Minimal access aortic valve replacement: is it worth it? Ann Thorac Surg 2008;85:1121-31.

18. Kirmani BH, Jones SG, Malaisrie SC, et al. Limited versus full sternotomy for aortic valve replacement. In Cochrane Database of Systematic Reviews. John Wiley \& Sons, Ltd. 2007.

19. Phan K, Xie A, Di Eusanio M, et al. A meta-analysis of minimally invasive versus conventional sternotomy for aortic valve replacement. Ann Thorac Surg 2014;98:1499-511.

20. Fattouch K, Moscarelli M, Del Giglio M, et al. Nonsutureless minimally invasive aortic valve replacement: mini-sternotomy versus mini-thoracotomy: a series of 1130 patients. Interact Cardiovasc Thorac Surg 2016;23:253-8.

21. Miceli A, Gilmanov D, Murzi M, et al. Minimally invasive aortic valve replacement with a sutureless valve through a right anterior mini-thoracotomy versus transcatheter aortic valve implantation in high-risk patients. Eur J Cardiothorac Surg 2016;49:960-5.

22. Santarpino G, Pfeiffer S, Jessl J, et al. Sutureless replacement versus transcatheter valve implantation in aortic valve stenosis: a propensity-matched analysis of 2 
strategies in high-risk patients. J Thorac Cardiovasc Surg 2014;147:561-7.

23. Terwelp MD, Thourani VH, Zhao Y, et al. Minimally Invasive Versus Transcatheter and Surgical Aortic Valve Replacement: A Propensity Matched Study. J Heart Valve Dis 2017;26:146-54.

24. Cao C, Ang SC, Indraratna P, et al. Systematic review and meta-analysis of transcatheter aortic valve implantation versus surgical aortic valve replacement for severe aortic stenosis. Ann Cardiothorac Surg 2013;2:10-23.

25. Kodali SK, Williams MR, Smith CR, et al. Two-Year Outcomes after Transcatheter or Surgical Aortic-Valve Replacement. N Engl J Med 2012; 366:1686-95.

26. Glauber M, Miceli A. Minimally invasive aortic valve replacement with sutureless valve is the appropriate treatment option for high-risk patients and the "real alternative" to transcatheter aortic valve implantation. J Thorac Cardiovasc Surg 2016;151:610-3.

27. Glauber M, Miceli A, Gilmanov D, et al. Right anterior minithoracotomy versus conventional aortic valve

Cite this article as: Hoffmann CT, Heiner JA, Nguyen TC. Review of minimal access versus transcatheter aortic valve replacement for patients with severe aortic stenosis. Ann Cardiothorac Surg 2017;6(5):498-503. doi: 10.21037/ acs.2017.09.02 replacement: A propensity score matched study. J Thorac Cardiovasc Surg 2013;145:1222-6.

28. Sharony R, Grossi EA, Saunders PC, et al. Minimally invasive aortic valve surgery in the elderly: a case-control study. Circulation 2003;108 Suppl 1:II43-7.

29. Bening C, Hamouda K, Oezkur M, et al. Rapid deployment valve system shortens operative times for aortic valve replacement through right anterior minithoracotomy. J Cardiothorac Surg 2017;12:27.

30. Martínez-Comendador J, Castaño M, Gualis J, et al. Sutureless aortic bioprosthesis. Interact Cardiovasc Thorac Surg 2017;25:114-21.

31. Reser D, Walser R, van Hemelrijk M, et al. Long-Term Outcomes after Minimally Invasive Aortic Valve Surgery through Right Anterior Minithoracotomy. Thorac Cardiovasc Surg 2017;65:191-7.

32. Reardon MJ, Van Mieghem NM, Popma JJ, et al. Surgical or Transcatheter Aortic-Valve Replacement in Intermediate-Risk Patients. N Engl J Med 2017;376:1321-31. 